\title{
Ultrafast Excited-State Energy Transfer in DTDCTB Dimers Embedded in a Crystal Environment: Quantum Dynamics with the Multilayer Multiconfigurational Time-Dependent Hartree Method
}

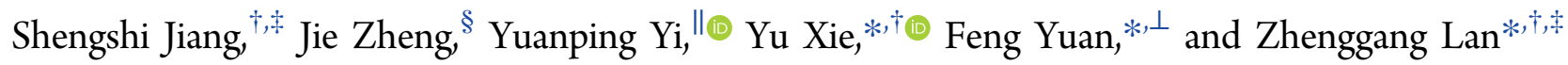 \\ ${ }^{\dagger}$ CAS Key Laboratory of Biobased Materials, Qingdao Institute of Bioenergy and Bioprocess Technology, Chinese Academy of \\ Sciences, Qingdao, 266101 Shandong China \\ ${ }^{\ddagger}$ University of Chinese Academy of Sciences, Beijing 100049, China \\ ${ }^{\S}$ Industrial Research Institute of Nonwovens \& Technical Textiles, College of Textiles \& Clothing, and ${ }^{\perp}$ College of Physics, Qingdao \\ University, Qingdao 266071, China \\ "Beijing National Laboratory for Molecular Sciences (BNLMS), CAS Key Laboratory of Organic Solids, Institute of Chemistry, \\ Chinese Academy of Sciences, Beijing 100190, China
}

Supporting Information

\begin{abstract}
Photoinduced excited-state energy transfer (EET) processes play a key role in the solar energy conversion of small molecule organic solar cells. We investigated intermolecular EET dynamics in the 2-[[7-(5- N,N-ditolylaminothiophen-2-yl)-2,1,3-benzothiadiazol-4-yl]methylene]malononitrile (DTDCTB) dimer embedded in a crystal environment using full quantum dynamics, i.e., the multilayer multiconfigurational time-dependent Hartree (ML-MCTDH) method. Two different stacking statuses of the DTDCTB dimers, which occur along the OA axis in the DTDCTB crystal, were considered. We built a vibronic diabatic Hamiltonian using the projection method based on quantum mechanics/molecular mechanics results. Different model Hamiltonians were considered in the full

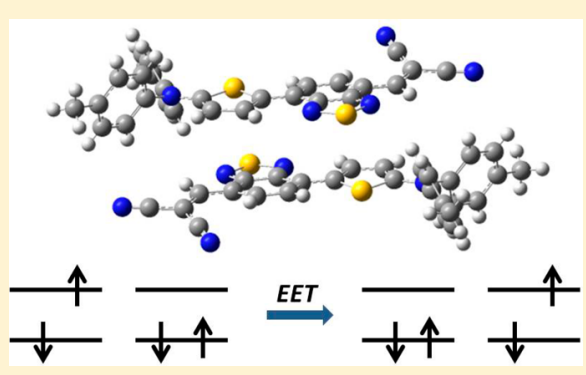
quantum dynamics studies. First, reduced-dimensional models were constructed by simply including more of the important vibrational modes. Second, we tried to construct a continuous spectral density based on the vibronic coupling strengths of different modes and then created a set of "pseudomodes" to represent electron-phonon couplings. The dynamics results based on these reduced models were compared with the results obtained with the full dimensional model. Our theoretical descriptions demonstrated that ultrafast intermolecular EET dynamics takes place in the well-stacked DTDCTB dimers. This work deepens our understanding of the photoinduced ultrafast EET dynamics of realistic organic photovoltaic systems at the full quantum mechanical level.
\end{abstract}

\section{INTRODUCTION}

Organic solar cells (OSCs) are promising alternatives for inorganic photovoltaic devices due to advantages such as flexibility, simple processing, and low cost. ${ }^{1}$ OSCs that consist of polymers and fullerene derivatives have achieved power conversion efficiencies (PCEs) of over $11 \%{ }^{2}$ In addition, PCEs of over $13 \%$ for OSCs composed of polymer-based donors and nonfullerene acceptors were reported recently. ${ }^{3}$ Alternatively, small molecule OSCs (SMOSCs) have also received substantial attention due to their well-defined molecular structures, easy purification, and better batch reproducibility compared to polymer-based OSCs. ${ }^{4,5}$ To date, PCEs of fullerene-based SMOSCs have already exceeded $11 \%{ }^{6}$ At the same time, the small molecules used in SMOSCs also provide good model systems for the theoretical study of the working mechanism of OSCs, because they do not display very complicated confirmations compared with long-chain conductor polymers.

The solar energy conversion of OSCs begins with photoabsorption, by which the excitonic states of donor materials are generated. Then, the excitons are transported to the donor- acceptor heterojunction interface and separated into weakly coupled hole-electron pairs. Therefore, exciton diffusion/ transfer is of great importance to the performance of OSCs. Recent time-resolved studies $^{7-10}$ determined that efficient charge generation may occur on a subpicosecond time scale. This readily infers that photoinduced exciton transport between donor compounds should also occur in an ultrafast time scale and perhaps within the subpicosecond domain. Many experimental investigations have confirmed that ultrafast excited-state energy transfers (EETs) occur in electron-donor materials in polymer-based OSCs ${ }^{11-14}$ and SMOSCs. ${ }^{15-17}$ Extensive theoretical efforts have also been undertaken to understand EET in OSCs. ${ }^{18-28}$

Recently, the 2-[[7-(5-N,N-ditolylaminothiophen-2-yl)-2,1,3benzothiadiazol-4-yl]methylene]malononitrile (DTDCTB) based OSCs have received great research interest due to their

Received: August 16, 2017

Revised: November 4, 2017

Published: November 14, 2017 


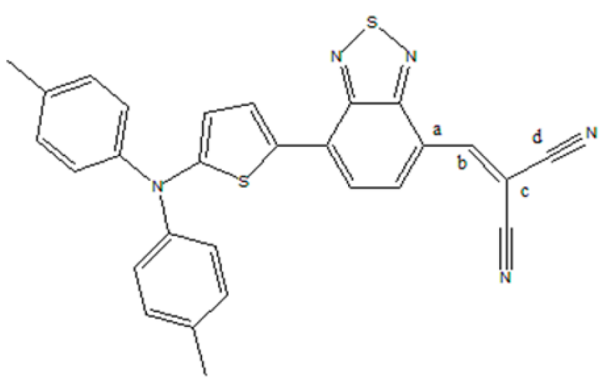

(a)

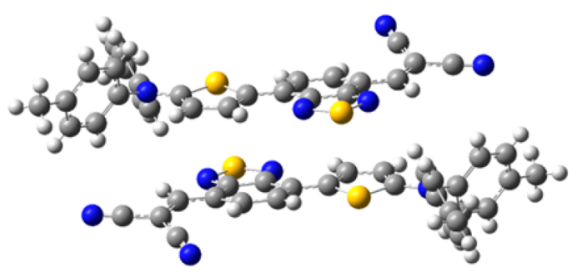

(c)

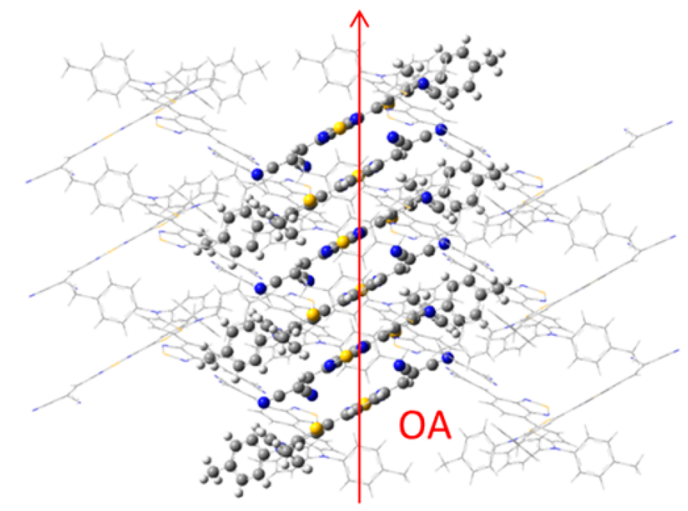

(b)

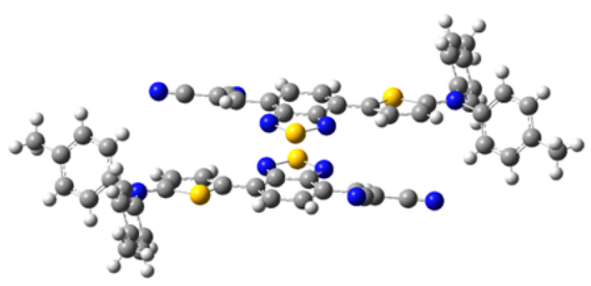

(d)

Figure 1. (a) Chemical structure of the DTDCTB molecule. (b) DTDCTB crystal structure and the OA axis. (c) Dimer I. (d) Dimer II.

relatively high PCEs. ${ }^{29-34}$ Time-resolved experiments have demonstrated that ultrafast charge separation occurs $(\sim 100 \mathrm{fs})$ in the DTDCTB/fullerene heterojunction films. ${ }^{30}$ This implies the occurrence of ultrafast EET among donor compounds after photoabsorption. Systematic theoretical studies on different aspects of DTDCTB-based SMOSCs have been performed. ${ }^{31-34}$ The calculations indicated that the transition densities of low-lying adiabatic excited states are delocalized over the entire stacked DTDCTB dimers/trimers, and such delocalization increases exciton diffusion and further facilitates ultrafast charge generation. ${ }^{32}$ Such delocalization also implies strong electronic couplings between different DTDCTB monomers, implying the strong possibility of ultrafast intermolecular EET dynamics in DTDCTB aggregates. The simulation of the morphology under different deposition growth conditions ${ }^{33}$ clarified that the crystal structure of DTDCTB is well maintained in the formation of DTDCTB/ fullerene heterojunctions. Overall, these works imply that ultrafast intermolecular EET may occur in well-stacked DTDCTB aggregates.

To provide some preliminary understanding of EET dynamics in DTDCTB aggregates, we may rationally start from the stacked dimer systems. The crystal environment may play an important role here. In this work, we investigated EET dynamics in DTDCTB dimers embedded in a crystal environment using quantum dynamics. Two different closely stacked DTDCTB dimers were considered, because they should represent the most efficient mechanisms for EET in the DTDCTB crystal. The diabatic model was constructed using the wave function projection method, and nuclear motions were described by normal mode coordinates. The parameters in the diabatic Hamiltonian were obtained by electronic structure calculations with the ONIOM approach. After the construction of a diabatic Hamiltonian, the multi- mode EET dynamics were studied by the multilayer multiconfigurational time dependent Hartree (ML-MCTDH) method. This work deepens our understanding of ultrafast EET dynamics in SMOSCs.

This article is organized as follows. In section 2, theoretical methods are introduced, including the construction of the diabatic EET Hamiltonian, electronic-structure calculations and quantum dynamics, etc. The results and a discussion are given in section 3 , followed by our conclusion and outlook in section 4.

\section{METHODS}

2.1. System. In this work, we investigated excited energy transfer in dimers composed of two closely stacked DTDCTB molecules embedded in a crystal. The chemical structure of DTDCTB is shown in Figure 1a. Although different aggregative statuses occur in the DTDCTB crystal, well-arranged $\pi-\pi$ stacking patterns are found along the OA axis, as shown in Figure 1b. In principle, EET along such a direction should be most efficient because of the closely packed face-to-face stacking status. For other aggregative statuses, the intermolecular distance is much longer, and thus EET dynamics should not be very efficient. In this work, we mainly focused on the ultrafast intermolecular EET dynamics of the $\pi-\pi$ stacking DTDCTB dimer along the OA axis.

The two different configurations of $\pi-\pi$ stacking DTDCTB dimers along the OA axis, shown in Figure $1 c$,d, are denoted dimer I and dimer II. In dimer I, the thiophene moiety of one monomer faces the benzothiadiazole moiety of the other monomer. In the crystal, the thiophene-benzothiadiazole separation measured by their interplane distance is approximately 3.31 A. In dimer II, only the benzothiadiazole moieties of the two monomers face each other, and their distance is a little larger, $3.38 \AA$. The isolated dimer geometries are quite 
different from those in the crystal. For example, a large deviation is obtained for the dihedral angle $\mathrm{C}(\mathrm{a})-\mathrm{C}(\mathrm{b})-\mathrm{C}(\mathrm{c})-$ $\mathrm{C}(\mathrm{d})\left(177^{\circ}\right.$ in the isolated dimer and $137^{\circ}$ in the crystal; chemical structure of the DTDCTB molecule with the four labeled $\mathrm{C}$ atoms is given in Figure 1a). Thus, in this work, we consider DTDCTB dimers embedded in a crystal environment.

2.2. Diabatic Hamiltonian. The diabatic Hamiltonian to describe EET transfer reads

$$
\begin{aligned}
& H^{(\mathrm{d})}=T_{\text {nuc }}+V_{\mathrm{el}}^{(\mathrm{d})} \\
& V_{\mathrm{el}}^{(\mathrm{d})}=\sum_{\alpha}|\alpha\rangle\left\langle\alpha\left|V_{\alpha}+\sum_{\alpha \neq \beta}\right| \alpha\right\rangle\langle\beta| V_{\alpha \beta}
\end{aligned}
$$

where $T_{\text {nuc }}$ denotes the kinetic energy of the nuclear degrees of freedom and $V_{\mathrm{el}}^{(\mathrm{d})}$ represents the electronic Hamiltonian. $V_{\alpha}$ denotes the energy of excited state $\alpha$, and the off-diagonal element $V_{\alpha \beta}$ characterizes the interstate coupling. In the diabatic representation, the electronic states $|\alpha\rangle$ correspond to the local excited state (LE) localized in one monomer of the DTDCTB dimer. To estimate electron-phonon couplings (vibronic couplings), small nuclear displacements were considered within the normal mode approximation. For simplicity, dimensionless normal coordinates $Q_{i}$ with associated frequencies $\omega_{i}$ were used to represent the diagonal elements of the diabatic Hamiltonian, namely

$$
V_{\alpha}=E_{\alpha}+\frac{1}{2} \sum_{i} \omega_{i} Q_{i}^{2}+\sum_{i} \kappa_{i}^{(\alpha)} Q_{i}
$$

where $E_{\alpha}$ are energies of the electronic states at the reference geometry. $\kappa_{i}^{(\alpha)}$ represent the first-order intrastate vibronic couplings. We neglect the frequency shift and the Dushinsky rotation in this work. The diabatic couplings are treated as constants, because this approximation should already capture the leading contribution of interstate couplings. The Hamiltonian model is a typical spin-boson one that was extensively studied by previous works in the framework of MCTDH. ${ }^{35-37}$

Because each LE state is on one monomer, each LE state is mostly affected by the local modes of that monomer. In addition, considering the symmetry of the dimer structures, we only use the normal mode of a monomer embedded in crystal to calculate $\kappa_{i}^{(\alpha)}$ of an LE state and use the same parameter to describe the vibronic coupling of the other LE state. This is equivalent to assuming that different LE states couple to different bath modes.

2.3. Determination of Parameters in the Diabatic Hamiltonian. The parameters were determined by the quasidiabatization scheme on the basis of electronic structure calculation data. Because a similar scheme has been applied successfully for charge and exciton transfers in organic solar cells, ${ }^{38-44}$ only a brief description is given here.

Adiabatic states $\left|\Psi_{j}^{(\mathrm{a})}\right\rangle$ can be expanded as a linear combination of the diabatic states $\left|\Psi_{i}^{(\mathrm{d})}\right\rangle$, i.e.

$$
\left|\Psi_{j}^{(\mathrm{a})}\right\rangle=\sum_{i}\left|\Psi_{i}^{(\mathrm{d})}\right\rangle t_{i j}
$$

The reference geometry is chosen where adiabatic and diabatic states are identical. The electronic states at the reference geometry are called reference states. By projecting adiabatic states onto reference states, we obtain the nonorthogonal adiabatic-diabatic transformation matrix $\tilde{\mathbf{T}}$ with elements $\widetilde{t}_{i j}$, i.e.

$$
\tilde{t}_{i j}=\left\langle\Psi_{i}^{(\mathrm{ref})} \mid \Psi_{j}^{(\mathrm{a})}\right\rangle
$$

where $\left|\Psi_{i}^{(\text {ref) })}\right\rangle$ denote the reference states. In practice, when we simply enlarge the intermolecular distance of an isolated dimer, the adiabatic wave functions are a good approximation to diabatic ones. $\tilde{\mathbf{T}}$ is orthogonalized using

$$
\mathbf{T}=\tilde{\mathbf{T}}\left(\tilde{\mathbf{T}}^{\dagger} \tilde{\mathbf{T}}\right)^{-1 / 2}
$$

where $\mathbf{T}$ is the orthogonal matrix with elements $t_{i j}$. Then, the diabatic Hamiltonian matrix can be written as

$$
\mathbf{V}_{\mathrm{el}}^{(\mathrm{d})}=\mathbf{T} \mathbf{V}_{\mathrm{el}}^{(\mathrm{a})} \mathbf{T}^{\dagger}
$$

where $\mathbf{V}_{\mathrm{el}}^{(\mathrm{d})}$ and $\mathbf{V}_{\mathrm{el}}^{(\mathrm{a})}$ are the diabatic and adiabatic electronic Hamiltonian matrices, respectively. Thus, $V_{\alpha}$ and $V_{\alpha \beta}$ were readily obtained from the diabatic electronic Hamiltonian matrix. $\kappa_{i}^{(\alpha)}$ were obtained from the gradients of the diabatic electronic states at the equilibrium geometry of the system.

2.4. Vibronic Couplings and Spectral Density. In the present work, two approaches were taken to represent the vibronic couplings.

The vibronic couplings can certainly be represented by the first-order intrastate couplings $\kappa_{i}^{(\alpha)}$ over a group of discretized normal modes. In the ML-MCTDH study, we may simply construct the diabatic vibronic Hamiltonian including all important normal modes. This directly provides a solution accessing the accuracy of the full-dimensional limit.

Alternatively, electron-phonon coupling may also be characterized by a continuous spectral density $J(\omega)$ constructed based on the $\kappa_{i}^{(\alpha)}$ of all modes. Previous works also largely employed this idea. ${ }^{39,45,46}$ In particular, this approach is very attractive because the continuous spectral density can help us to build the equation of motion for quantum dissipative dynamics based on the density operator and system-bath model. ${ }^{47,48}$ This approach provides an alternative way to solve the quantum dynamics of large systems with many degrees of freedom.

The spectral density was built as

$$
\begin{aligned}
J(\omega) & =\frac{\pi}{2} \sum_{i=1}^{N_{0}} \kappa_{i}^{2} \delta\left(\omega-\omega_{i}\right) \\
& \approx \frac{\pi}{2} \sum_{i=1}^{N_{0}} \frac{\kappa_{i}^{2}}{\pi} \frac{\Delta}{\left(\omega-\omega_{i}\right)^{2}+\Delta^{2}}
\end{aligned}
$$

where $\Delta$ is the width of the Lorentzian function and $N_{0}$ is the number of normal modes.

Following the suggestion in previous works, ${ }^{39}$ the value of $\Delta$ is taken as the root-mean-square (RMS) of the frequency spacing of all modes. We chose two values for $\Delta\left(\Delta_{1}\right.$ and $\left.\Delta_{2}\right)$ in the construction of the spectral density. The first value $\Delta_{1}=$ $0.010 \mathrm{eV}$ is very close to the RMS of the frequency spacing of all modes. Because there are only a small number of normal modes in the frequency region of $\omega>1800 \mathrm{~cm}^{-1}$ and most of them show very minor vibronic couplings, we also computed the second value $\Delta_{2}=0.002 \mathrm{eV}$ close to the RMS of the frequency spacing without considering the modes in the highfrequency $\left(\omega>1800 \mathrm{~cm}^{-1}\right)$ domain.

Starting from the continuous spectral density, we constructed a group of pseudo phonon modes with an arbitrary number of modes $N$. The electron-phonon coupling $\kappa_{\mathrm{ps}, i}$ of each pseudomode with frequency $\omega_{\mathrm{ps}, i}$ is given by

$$
\kappa_{\mathrm{ps}, i}=\left(\frac{2}{\pi} J\left(\omega_{\mathrm{ps}, i}\right) \Delta \omega_{\mathrm{ps}}\right)^{1 / 2}
$$


where $\Delta \omega_{\mathrm{ps}}$ is the frequency interval between two adjacent pseudomodes, which defines the Poincare recurrence period $\tau_{\mathrm{p}}$ $=2 \pi / \Delta \omega_{\mathrm{ps}}$. For $t<\tau_{\mathrm{p}}$, the observed dynamics are effectively irreversible. Thus, we map the original multimode Hamiltonian to a new diabatic Hamiltonian including many pseudomodes with equal frequency spacing. For illustrative purpose, we will use "modes" to represent the normal modes or vibrational modes in the below discussion, whereas the expression "pseudomodes" refers to pseudomodes constructed from discretization of a continuous spectral density.

2.5. Quantum Dynamics. 2.5.1. ML-MCTDH. In this work, we take a powerful extension of the usual MCTDH approach, ${ }^{49}$ ML-MCTDH, ${ }^{50-56}$ to simulate the quantum dynamics. As a rigorous variational approach, ML-MCTDH can give accurate solutions of full quantum dynamics for large systems with thousands of degrees of freedom. ${ }^{53,54}$ The original MCTDH equation of motion is

$$
\Psi\left(Q_{1}, \ldots, Q_{f}, t\right)=\sum_{j_{1}=1}^{n_{1}} \ldots \sum_{j_{f}=1}^{n_{f}} A_{j_{1}, \ldots, j_{f}}(t) \prod_{\kappa=1}^{f} \varphi_{j_{\kappa}}^{(\kappa)}\left(Q_{\kappa}, t\right)
$$

where $Q_{1}, \ldots, Q_{f}$ are the degrees of freedom (DOFs). $A_{j, \ldots, j_{f}}$ denote the time-dependent expansion coefficients. $\varphi_{j_{k}}^{(\kappa)}$ denote the time-dependent basis functions that are called single particle functions (SPFs). The structure of the equations of motion (EOMs) of ML-MCTDH is very similar to the usual MCTDH equations, whereas the SPFs in ML-MCTDH are expanded recursively as a combination of the time-dependent basis functions. The recursive expansion is

$$
\begin{aligned}
& \varphi_{m}^{l-1 ; \kappa_{1}, \ldots, \kappa_{l-1}}\left(Q_{\kappa_{l-1}}^{l-1 ; \kappa_{1}, \ldots, \kappa_{l-2}}, t\right) \\
& =\sum_{j_{1}=1}^{n_{1}} \ldots \sum_{j_{p \kappa_{l}}=1}^{n_{\kappa_{l}}} A_{m ; j_{1}, \ldots, j_{p \kappa_{l}}}^{l ; \kappa_{1}, \ldots, \kappa_{l-1}}(t) \prod_{\kappa_{l}=1}^{p_{\kappa_{l}}} \varphi_{\kappa_{l}}^{l ; \kappa_{1}, \ldots, \kappa_{l}}\left(Q_{\kappa_{l}}^{\left.l ; \kappa_{1}, \ldots, \kappa_{l-1}, t\right)}\right.
\end{aligned}
$$

where $l$ denotes the layer depth; $\kappa_{1}, \ldots, \kappa_{l-1}$ denote the indices of the logical degrees of freedom starting from each node on the top layer down to a particular primary coordinate. Therefore, a tree structure can be constructed to represent the wave function in ML-MCTDH. The EOMs can be obtained applying the recursive algorithm provided by Manthe. ${ }^{55,56}$ Essentially, the ML-MCTDH is much more powerful due to its arbitrary layering schemes, which is by nature a tensor decomposition method. All ML-MCTDH calculations in this work are performed using the Heidelberg MCTDH package. ${ }^{57}$

2.5.2. Initial Condition. The initial wave packet $\psi(t=0)$ is obtained by vertical excitation of the ground vibrational level of the electronic ground state to a local excited state $\left(\mathrm{LE}_{1}\right)$ on one subunit of a DTDCTB dimer according to the Condon approximation. The diabatic population and the electronic coherence are represented by the diagonal elements $P_{i, i}(t)$ and the off-diagonal elements $P_{i, j}(t)$ of the reduced electronic density matrix, i.e.

$$
P_{i, j}(t)=\operatorname{Tr}_{b}\left\{\left|\phi_{j}\right\rangle\left\langle\phi_{i}\right| \hat{\rho}(t)\right\}=\int \rho_{i, j}(Q, t) \mathrm{d} Q
$$

where the density operator is defined as $\hat{\rho}=|\psi(t)\rangle\langle\psi(t)|$ and the trace $\left(\operatorname{Tr}_{b}\right)$ is over all vibrational degrees of freedom.

2.6. Electronic Structure Calculation Details. All electronic structure calculations are performed using the Gaussian 09 package. The calculations of the electronic ground state and excited states were performed based on the ONIOM approach. A two-layer model was adopted for the ONIOM calculations. The high layer was treated by density functional theory (DFT) and time-dependent DFT (TDDFT). The longrange correction functional CAM-B3LYP and the 6-31G* basis set were used in DFT and TDDFT calculations. The DTDCTB dimer was chosen as the high layer in the excited state calculations. The environment composed of adjacent DTDCTB compounds was treated as the low layer described by the universal force field (UFF). The geometry of the low layer was fixed in the calculations.

To perform the diabatization procedure, the reference geometry defined in section 2.3 was chosen by simply taking the structure of the environment-free or isolated dimer with a very large intermolecular distance. Then, excited state calculations of the reference geometry were performed using TDDFT at the same level that was used in the high layer of the ONIOM calculations of the embedded system.

Due to the symmetry of the two dimer structures, the normal mode calculations were performed only by considering the DTDCTB monomer embedded in the environment. By such a construction, we can take all parameters obtained from electronic structure calculation and diabatization, simply include more compounds to build the diabatic model of DTDCTB aggregates, and then study EET in more complicated systems.

\section{RESULTS AND DISCUSSION}

3.1. Characterization of the System. In this work, we perform a quantum dynamics investigation on the EET dynamics of DTDCTB dimers embedded in a crystal environment. Two typical DTDCTB dimers, dimer I and dimer II [along the OA axis of the crystal in Figure 1], are considered. The two dimer structures of the ground state energy minimum geometry are nearly identical to the original structures in the primitive crystal.

The electronic state site energies and the intersite couplings in the diabatic Hamiltonian are given in Table 1. The computed

Table 1. Elements of the Electronic Hamiltonian Matrix of the Two Typical Dimers, i.e., Energies $E_{\alpha}$ of the Two States and Their Couplings $V_{\alpha \beta}(\text { in } \mathrm{eV})^{a}$

$\begin{array}{cccc}\text { dimer } & E_{\mathrm{LE}_{1}} & E_{\mathrm{LE}_{2}} & V_{\mathrm{LE}_{1} \mathrm{LE}_{2}} \\ \text { I } & 2.354 & 2.354 & 0.136 \\ \text { II } & 2.354 & 2.354 & 0.091\end{array}$

${ }^{a} \mathrm{LE}$ represents the local excited state.

site energies of LE states in the two dimers are all identical, which is in accordance with the localized properties of LE states. The intersite electronic coupling in dimer I is larger than that of dimer II, because dimer I has a smaller interplane distance and more $\pi-\pi$ overlap between monomers.

3.2. Vibronic Coupling. The contribution from the vibronic coupling of each mode to EET is characterized by a shift of the potential minimum, $\Delta x_{i}$, along each mode

$$
\Delta x_{i}=\frac{\left|\kappa_{i}^{(\alpha)}\right|}{\omega_{i}}
$$

which is given in Figure 3a. There are two important modes with $\Delta x_{i}>0.6$ near $1500 \mathrm{~cm}^{-1}$. This region corresponds to the in-plane ring deformation of ring moieties (Figure 2) that plays 


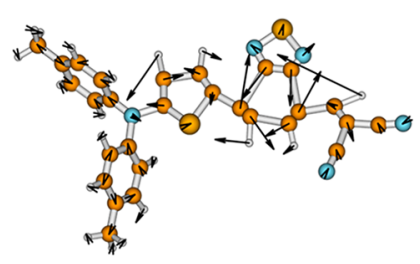

$1424 \mathrm{~cm}^{-1}$

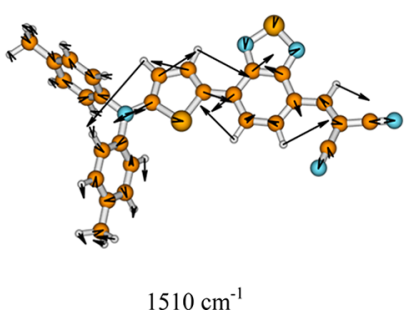

Figure 2. Views of the two most important vibrational modes.

a major role in EET. In the frequency domain from 200 to 1500 $\mathrm{cm}^{-1}$, a few modes display visible electron-phonon couplings of $\Delta x_{i}>0.3$, which should be considered in the treatment of EET dynamics. Most modes with frequencies larger than 1800 $\mathrm{cm}^{-1}$ show very minor electron-phonon couplings. Only two modes relevant to $\mathrm{C} \equiv \mathrm{N}$ bond stretching motions show small couplings $\left(\Delta x_{i}>0.1\right)$. Each of these relevant modes is given in Appendix I.

It is well-known that a low-frequency mode is not well described by the normal mode approximation. ${ }^{58}$ In addition, because the system is embedded in the environment, the normal modes are constructed using a partial Hessian with fixed environmental atomic positions. Thus, the low-frequency modes are not reliable. Our preliminary calculations indicate that the pure electronic dynamics governed by electronic coupling $V_{12}$ in the present systems are much faster than the period of the vibrational mode with low frequency of $\sim 200$ $\mathrm{cm}^{-1}$. Previous works indicated that the influence of lowfrequency modes on ultrafast nonadiabatic dynamics is very minor due to their different time scales. ${ }^{59,60}$ Thus, we do not consider the low-frequency mode in the dynamics calculations.

The continuous spectral densities $J(\omega)$ without the inclusion of low-frequency modes $\left(\omega \leq 200 \mathrm{~cm}^{-1}\right)$ are shown in Figure 3b. The curve with $\Delta_{1}=0.010 \mathrm{eV}$ displays a smooth feature with one big peak at approximately $1500 \mathrm{~cm}^{-1}$, whereas the one with a smaller $\Delta\left(\Delta_{2}=0.002 \mathrm{eV}\right)$ shows fine features with several distinct peaks. The continuous spectral densities are rediscretized to give pseudo phonon modes for the different mode numbers $(N=19,57,76)$ associated with different $\Delta \omega$ values $[\Delta \omega=0.02,0.0066,0.005$ (unit $=\mathrm{eV}$ ) ]. Because we use the normal mode of the monomer to set up the vibronic couplings, the total number of modes in the dimer should be $2 N$. In total, 38,114 , and 152 pseudomodes were involved in the ML-MCTDH calculations.

3.3. EET Dynamics. We simulated the ultrafast EET dynamics of the DTDCTB dimer embedded in a crystal using ML-MCTDH. In the first step, the most intuitive way was considered. We tried to perform the MCTDH calculation on the reduced model system by including several important modes characterized by large $\Delta x_{i}$. We included more and more modes into the model Hamiltonian according to a different cutoff of $\Delta x_{i}$, until finally the full-dimensional model (without modes with extremely low frequencies) was treated explicitly. We need to indicate that the time scales of many important modes, such as modes near $1400-1700 \mathrm{~cm}^{-1}$, are comparable to that of the pure electronic motion with Rabi oscillations. Thus, the resonance situation should be automatically considered by the present approaches. The results with different numbers of important modes are shown in Figure 4a. For convenience, a reduced model with $n$ important modes is labeled R-1- $n$ below. In all cases, a rapid decay and strong recurrence of the $L E_{1}$ population is observed in the very early stage of the EET dynamics. Such a large oscillation is partially suppressed due to vibronic couplings, when dozens of modes (for instance 44 modes, R-1-44) are included. Such damping effects become more pronounced with more modes (R-1-60, 60 modes). When enough modes (R-1-98, 98 modes) are included, the time-dependent population starts to converge to the quantum dynamics results of the full dimensional model. Overall, the inclusion of vibrational modes provides damping effects, eliminating the forward-and-backward EET and stabilizing the electronic population. When enough modes are considered, we should obtain the accurate and converged EET dynamics.

An alternative way to build the reduced model is to discretize the continuous spectral density $J(\omega)$. For convenience, we use R-2- $\Delta_{i}-n$ to label such a reduced model, in which the continuous bath spectral density is constructed with boarding parameter $\Delta_{i}$ from discretized modes and then $n$ pseudomodes are generated by discretizing this spectral density. The MLMCTDH results of the R-2- $\Delta_{1}-38$, R-2- $\Delta_{1}-114$, and R-2- $\Delta_{1}-152$ models are shown in Figure $4 \mathrm{~b}$. All cases give a very similar feature: the early fast oscillation of electronic dynamics, and then the decrease of this oscillation and final population stabilization. In particular, all models give almost identical results for the dynamics with $t<100$ fs. However, a weak fluctuation of the electronic population occurs after $t>100 \mathrm{fs}$ for R-2- $\Delta_{1}-38$, whereas this feature almost disappears completely for R-2- $\Delta_{1}-114$ and R-2- $\Delta_{1}-152$. A similar tendency is also observed with $\Delta_{2}$, except that the population fluctuation becomes slightly stronger due to the smaller value of $\Delta_{2}$. Although all models with different numbers of pseudomodes show very similar results within a short time, the R-2- $\Delta_{2}-38$ model gives different dynamics in the long-time simulation. As shown in Appendix II, significant population recurrences occur
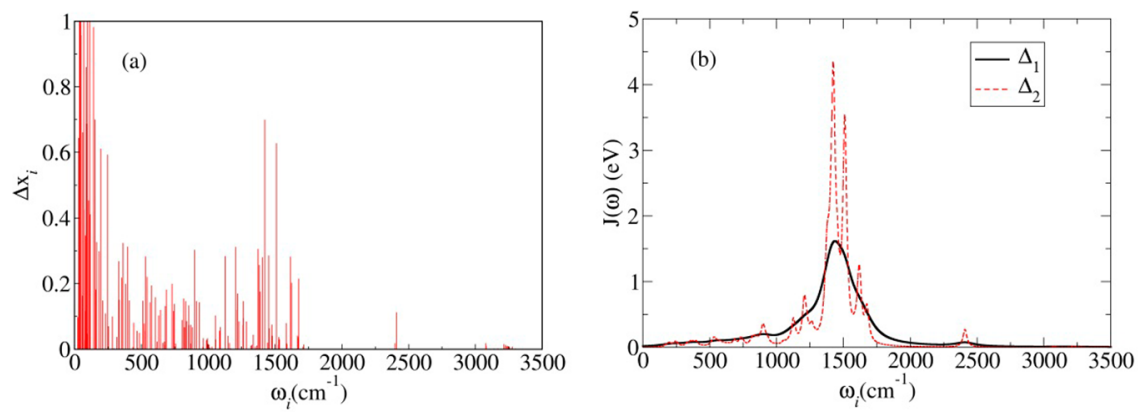

Figure 3. (a) Shift of potential minima, $\Delta x_{i}$, for all normal modes of the DTDCTB monomer embedded in a crystal. (b) Continuous spectral density $J(\omega)$ with two $\Delta$ 's $\left(\Delta_{1}=0.010 \mathrm{eV}\right.$ and $\left.\Delta_{2}=0.002 \mathrm{eV}\right)$. 

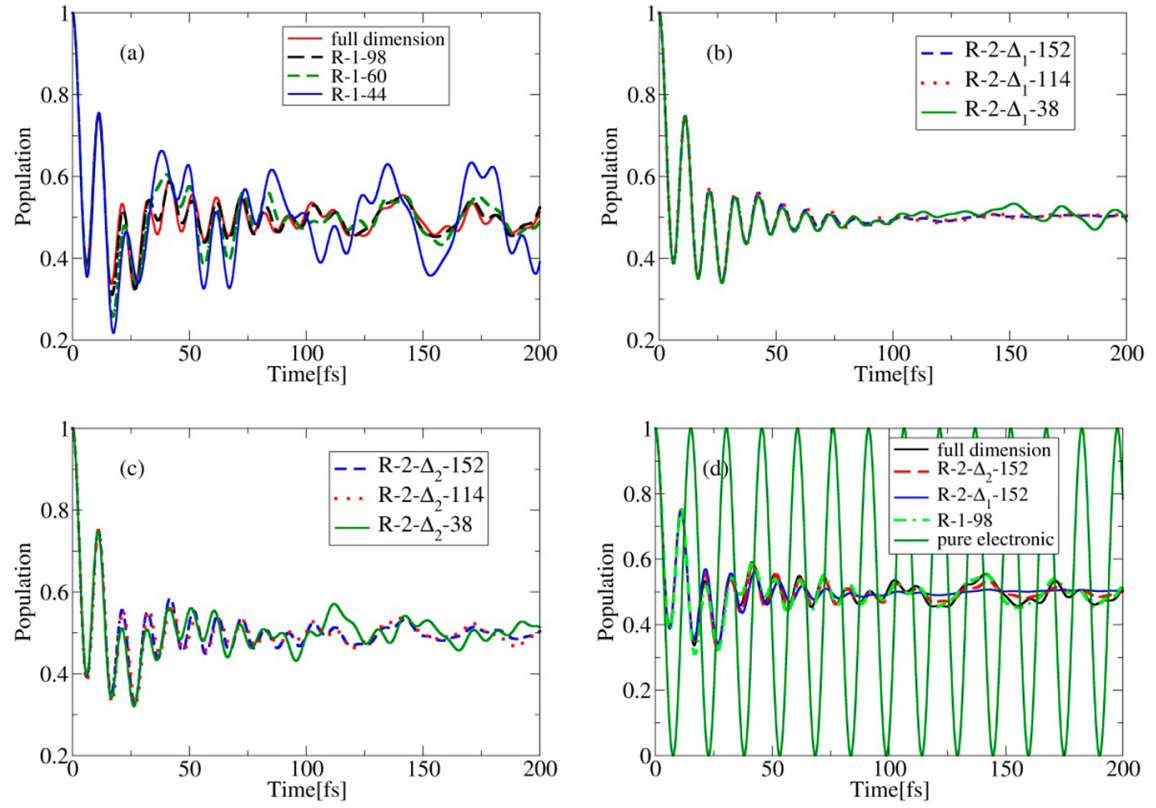

Figure 4. (a) LE $\mathrm{E}_{1}$ population in EET dynamics of dimer I including different numbers of important modes: R-1-44, R-1-66, R-1-98, and the full dimensional model. (b) Population of $\mathrm{LE}_{1}$ in the dynamics for dimer I using $\Delta_{1}$ with different numbers of pseudomodes: R-2- $\Delta_{1}-38$, R-2- $\Delta_{1}-114$, and R-2- $\Delta_{1}-152$. (c) Population of $\mathrm{LE}_{1}$ of dimer I in the dynamics for dimer I using $\Delta_{2}$ with different numbers of pseudomodes: R-2- $\Delta_{2}-38, \mathrm{R}-2-\Delta_{2}-$ 114, and R-2- $\Delta_{2}-152$. (d) Population of $\mathrm{LE}_{1}$ in the dynamics of dimer I for the pure electronic state, R-1-98, R-2- $\Delta_{1}-152, \mathrm{R}-2-\Delta_{2}-152$, and full dimension (278 modes) cases.
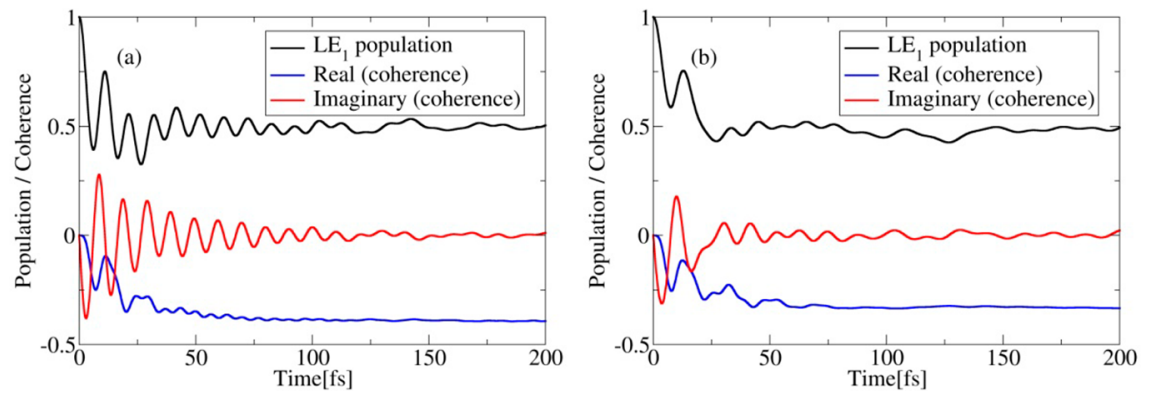

Figure 5. Population of the $\mathrm{LE}_{1}$ state and the real and imaginary parts of the electronic coherence of the R-2- $\Delta_{2}-152$ model for dimer I (a) and dimer II (b).

in both 38-mode cases, regardless of whether the continuous spectral density is constructed from $\Delta_{1}$ or $\Delta_{2}$, possibly due to the Poincaré recurrence.

A comparison between the dynamics of the pure electronic state, different reduced models (R-1-98, R-2- $\Delta_{1}-152$, and R-2$\left.\Delta_{2}-152\right)$, and full dimensional models is made in Figure $4 \mathrm{~d}$. As expected, the pure electronic dynamics gives a perfect Rabi oscillation. The rapid oscillation of electronic population is eliminated quickly by the inclusion of dozens of vibrational modes. Most cases give results very similar to those of the full dimensional model, whereas the broader and smooth spectral density seems to eliminate the population fluctuation completely after $100 \mathrm{fs}$. The main population features of the important-mode approach and the pseudomode approach are similar. There are still differences between the two methods. As shown in Figure 4a, in the important-mode approach, the result of the reduced model with 98 modes correctly reproduces the results of the model with all modes ( 246 modes). This implies that the model with 98 modes is enough in calculations. In the important-mode approach, the relevant modes are not equally distributed in the frequency domain. However, in the pseudomode approach, such as the R-2- $\Delta_{2}-152$ case, the sampled frequency spacing is equal. In the population dynamics, the latter approach seems to induce a slightly stronger damping effect and to weaken the population oscillation from 100 to $200 \mathrm{fs}$. In this case, the frequency spacing is equally small and every two neighbor modes should have very similar frequencies and couplings. The energy redistribution over these modes may become more effective. This may explain why the damping effect becomes a little stronger. However, such minor difference does not modify the main feature of the nonadiabatic dynamics. Thus, both the important-mode approach and the pseudomode approach can be employed to study this type of nonadiabatic dynamics, when the continuous spectral density and pseudomodes are properly constructed.

We tried to perform the dynamical calculation of the R-2- $\Delta_{2}$ 114 model at $T=300 \mathrm{~K}$ by the thermal weighted average of several dynamical results starting from randomly chosen initial conditions. As shown in Figure S1 in the Supporting Information (SI), temperature has little effect on EET dynamics. The reason is as follow. The current EET dynamics takes place in the ultrafast time domain $(<100 \mathrm{fs})$. At $T=300$ $\mathrm{K}$, only the low-frequency modes with very slow motion may be 
easily excited, while their time scale is slower compared with the ultrafast electronic motions. The modes with extremely low frequencies $\left(<200 \mathrm{~cm}^{-1}\right)$ are not considered in our model. Except for them, the modes with frequencies less than 800 $\mathrm{cm}^{-1}$ display very weak vibronic couplings. Overall, the thermal excitation of the low-frequency modes should have rather less contribution to the current ultrafast nonadiabatic dynamics.

Although the low-frequency modes only have minor dynamical effects here, they can play some roles in the realistic systems due to the static effects. The motion of low-frequency vibrational modes may result in different stacking geometries instantaneously. Also, due to their very slow motion, we can assume that the very fast EET dynamics happens at these instantaneous geometries by freezing the low-frequency motions. The discussion of this static disorder is very challenging, which is beyond the scope of the current work. Overall, the low-frequency modes might have a potential influence on the EET dynamics, when the electronic dynamics occur within a similar time scale to the vibrational motions or the static disorder is considered.

The $\mathrm{LE}_{1}$ population and $\mathrm{LE}_{1} / \mathrm{LE}_{2}$ electronic coherence of dimer I in the R-2- $\Delta_{2}-152$ case are given in Figure $5 \mathrm{a}$. The real and imaginary parts of the electronic coherence display an obvious oscillation in the early stage of EET dynamics (within $40 \mathrm{fs}$ ), which is consistent with the time-dependent $\mathrm{LE}_{1}$ state population feature. The real part becomes nearly flat, and the imaginary part displays weak oscillation after $\sim 70 \mathrm{fs}$. This result indicates that the weak recurrence of electronic population with time is in accordance with the residual $\mathrm{LE}_{1} / \mathrm{LE}_{2}$ electronic coherence. After $120 \mathrm{fs}$, the imaginary term decays completely to zero and the real part holds a constant value, -0.4 . As a result, the $\mathrm{LE}_{1}$ population starts to stabilize near 0.5. Thus, it is clear that the ultrafast EET dynamics is almost completely governed by the electronic coherence. The introduction of vibrational modes brings significant damping effects that quickly eliminate the electronic coherence oscillation and further stabilize the electronic population.

The only difference between the Hamiltonian models of dimer I and dimer II is that the latter displays a smaller electronic coupling value. We performed ML-MCTDH calculations on dimer II using the convergence conditions of dimer I (R-2- $\left.\Delta_{2}-152\right)$. The dynamics are rather similar, and thus we only summarize the main results in Figure $5 \mathrm{~b}$. Because of the smaller coupling of the two LE states in dimer II, the damping effect by the vibrational modes is more dramatic and the dynamics have weaker oscillation features (oscillation period and amplitude) than dimer I. As expected, the EET dynamics of dimer II are also relevant to electronic coherence.

It is possible to provide further insight into the present EET dynamics based on an analysis of the time scales of electronic and nuclear motion. Taking dimer $\mathrm{I}$ as an example, the electronic coupling $V_{\alpha \beta}$ is $0.136 \mathrm{eV}$. According to the vibronic couplings of all vibrational modes, we noticed that two types of modes give an important contribution to the present EET processes. The first type of important mode is the lowfrequency mode with the parameter $V_{\alpha \beta} / \omega_{\mathrm{c}} \gg 1$, which defines a slow bath with respect to the fast electronic motion. ${ }^{36}$ Previous works demonstrated that ultrafast dynamics are not influenced by these low-frequency modes. ${ }^{59,60}$ The second type of important mode shows that $V_{\alpha \beta}$ and $\omega_{\mathrm{c}}$ are comparable, and thus the electronic and vibrational dynamics fall into the same time scale. ${ }^{36}$ This indicates that the present EET dynamics are essentially non-Markovian, and thus ML-MCTDH is the preferred method for obtaining the precise theoretical treatment.

The DTDCTB molecule is one of the efficient donors in small molecule organic solar cells (SMOSCs). The understanding of exciton dynamics not only explains the physical insight into photoinduced reaction mechanisms, but also provides some ideas of rational design of organic photovoltaic compounds. In organic solar cells, the high energy conversion efficiency indicates the efficient charge separation at donoracceptor bulk heterojunctions, while the fast exciton transport to donor/acceptor interfaces is a prerequisite for such a step. Previous works suggested that the low-lying adiabatic excited states of stacked DTDCTB dimers/trimmers are delocalized over the entire systems, and such delocalization increases exciton diffusion and further facilitates ultrafast charge generation. ${ }^{32}$ However, it is very challenging to study the nonadiabatic dynamics of these realistic systems, because the whole systems include several donor/acceptor compounds and a huge number of nuclear nuclear degrees of freedom. To conquer this problem step by step, we first studied the EET dynamics in the DTDCTB dimers embedded in crystal. Although the current model systems are rather simple, several interesting conclusions can be drawn from this work:

1. Interestingly, we found the EET dynamics is ultrafast $(<100 \mathrm{fs})$. This implies that the exciton energy transfer to the interface is indeed very fast, giving a high chance to result in the efficient charge separation.

2. Electronic coherence maintains in a very long time range. Before the vanishing of electronic coherence, the exciton should be delocalized over several donor molecules. If the charge-transfer dynamics takes place within such a time scale, we should consider the EET/ET mixed dynamics for the proper treatment of the exciton dynamics.

3. Several modes in the frequency region from 1300 to 1700 $\mathrm{cm}^{-1}$ strongly couple to electronic states. These modes correspond to the double-bond stretching motions in rings. In current conjugated systems, these vibrations change the bond lengths of the conjugated ring, which influence the energies of the $\pi$-bonds. Because both LE and charge-transfer (CT) excitation are related to the $\pi$-bonds of the ring, these modes display strong intrastate vibronic coupling on the $\mathrm{LE}$ and CT states. When the electronic dynamics takes place in a similar time scale as the vibrational motions of these modes, the efficient EET dynamics should be achieved. As we know, the time scale of pure electronic motion is determined by energy gaps and electronic couplings. Thus, if these properties are adjusted by rational design, we may get the efficient EET dynamics that is the prerequisite of efficient solar energy conversion.

Beside the above understandings, we also noticed the following issues:

1. A large geometry difference exists between the embedding DTDCTB geometry in crystal and its isolated geometry. For example, a large deviation is obtained for the dihedral angle $\mathrm{C}(\mathrm{a})-\mathrm{C}(\mathrm{b})-\mathrm{C}(\mathrm{c})-\mathrm{C}(\mathrm{d})\left(177^{\circ}\right.$ in the isolated dimer and $137^{\circ}$ in the crystal; the chemical structure of the DTDCTB molecule with the four $\mathrm{C}$ atoms labeled is given in Figure 1a). Thus, it is highly preferable to include crystal environments in the theoretical treatment of these systems.

2. Only a few modes are very important in the ultrafast EET dynamics. As discussed above, the study of the nonadiabatic dynamics in the realistic situations should include several donor/acceptor compounds and a huge number of nuclear 
degrees of freedom. The accurate treatment of the nonadiabatic dynamics of these realistic systems with ML-MCTDH becomes extremely challenging. Thus, it is necessary to build reduced models with less effective degrees of freedom, which capture the main feature of nonadiabatic dynamics of complicated systems. Because the current work pointed out which modes are important, we can use this information to build the reduced model and run ML-MCTDH calculations for the investigation of realistic complicated systems. This is a very interesting topic for the future.

\section{CONCLUSIONS}

In this work, we investigated the ultrafast EET dynamics of DTDCTB dimers in a crystal environment using the MLMCTDH quantum wave packet method. The DTDCTB dimers have two different $\pi-\pi$ stacking geometries along the OA axis in the DTDCTB crystal. The electronic excited state calculations of the DTDCTB dimers were performed with the ONIOM approach, in which the DTDCTB dimers were treated at the CAM-B3LYP/6-31G* level and the crystal environment was treated at the UFF level. We built the diabatic vibronic Hamiltonian using the wave function projection method. Two types of vibronic coupling models were constructed. In the first model, the diabatic Hamiltonian includes several important modes characterized by large first-order intrastate coupling strengths. In the second model, we first construct the continuous spectral density based on the ab initio results and then discretize the spectral density to define several effective pseudomodes. The quantum dynamics show consistent results in both approaches. The pure electronic EET dynamics exhibit Rabi-type oscillations in population dynamics and strong electronic coherence. After the inclusion of nuclear motion, strong quenching of coherence oscillation was observed, and ultrafast irreversible EET dynamics were obtained. We also noticed that a population recurrence appears in the long-time evolution if not enough modes are included.

The present way of constructing the vibronic diabatic Hamiltonian also allows us to build a Hamiltonian including LE and CT states on the same footing. This opens a possibility for studying the influence of the CT state on the ultrafast EET processes of organic photovoltaic systems with quantum dynamics. In addition, it is also possible to extend the current diabatization approach to construct the vibronic diabatic Hamiltonian of large aggregate models. For example, the parameters of various dimers with different stacked geometries are easily obtained using the same procedure, and then it is possible to combine these parameters to build the diabatic Hamiltonian of the large aggregate models. This provides a starting point for understanding the EET dynamics in more complex aggregates with full quantum dynamics. These topics will be the research subjects of our further work.

\section{APPENDIX}

\section{Deformations of Vibrational Modes}

In the frequency domain from 200 to $1500 \mathrm{~cm}^{-1}$, the modes with visible electron-phonon couplings $\left(\Delta x_{i}>0.3\right)$ are given in Figure 6.

Most modes with a frequency larger than $1800 \mathrm{~cm}^{-1}$ show very minor electron-phonon couplings. Only two modes relevant to the $\mathrm{C} \equiv \mathrm{N}$ bond stretching motions show small couplings $\left(\Delta x_{i}>0.1\right)$, which are also given in Figure 6 .
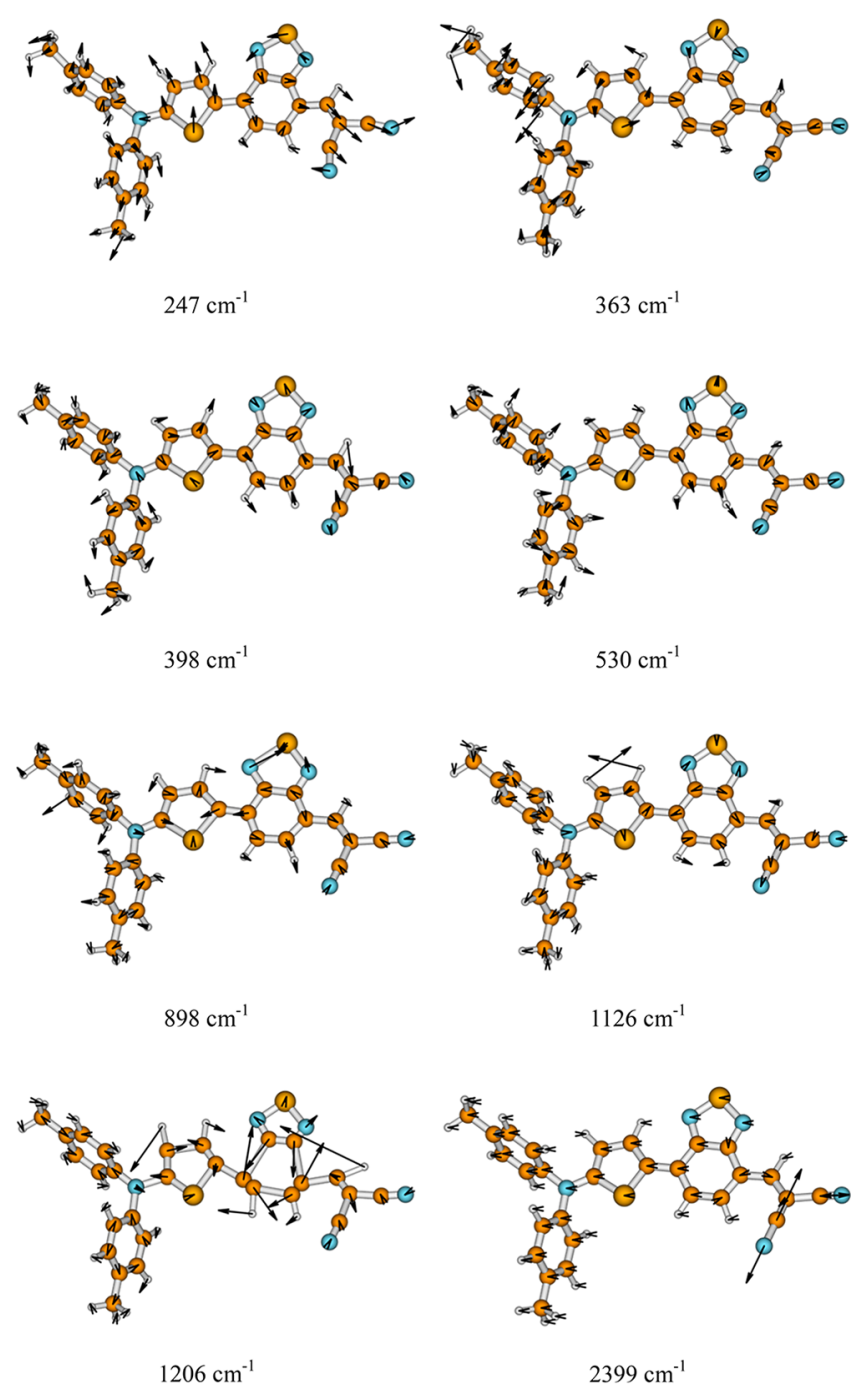

$1206 \mathrm{~cm}^{-1}$

$2399 \mathrm{~cm}^{-1}$

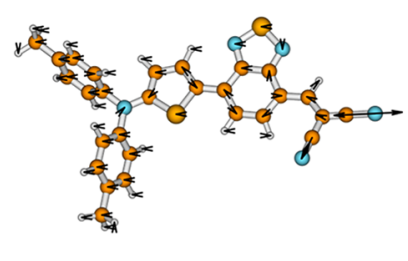

$2409 \mathrm{~cm}^{-1}$

Figure 6. Views of relevant vibrational modes.

\section{Long-Time Dynamics}

Long-time-dynamics simulations for $\Delta_{1}$ and $\Delta_{2}$ cases were also performed, and the results are shown in Figure 7. The Poincare recurrence period $\tau_{\mathrm{p}}$ of 114 and 152 pseudomodes are 631.5 and $833.6 \mathrm{fs}$, respectively. These results are longer than the simulation time scale of the present EET dynamics, so the dynamics may be viewed as an irreversible process. However, Figure 7 shows that the 38-mode models give the recurrence of the electronic population at $\sim 200 \mathrm{fs}$, because the Poincare recurrence period computed by this model is close to $208.4 \mathrm{fs}$.

\section{Optimal Construction of the ML-MCTDH Tree and Convergence Tests}

The construction of a reasonable wave function expansion tree is critical for the computational efficiency and convergence in the ML-MCTDH calculations. Previous studies provided some 

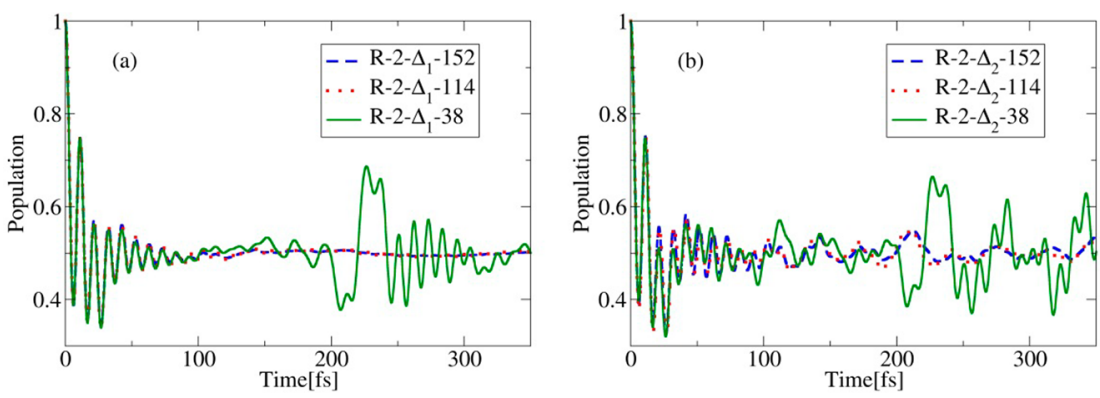

Figure 7. (a) $\mathrm{LE}_{1}$ population in the EET dynamics using $\Delta_{1}$ and different numbers of modes. (b) $L E_{1}$ population of the dynamics using $\Delta_{2}$ and different numbers of modes. The Poincare recurrence period of 38 pseudomodes is obvious for the two cases.

useful principles for this purpose, ${ }^{21,51,59,60}$ which gives us some hints to build tree expansion.

In the current work, several principles were employed to build the tree:

1. At the bottom level, we always tried to take the modes with similar frequencies and similar vibronic couplings to form a branch. When we combined two lower child branches into an upper parent branch, two child branches were also composed of the modes with similar frequencies and comparable vibronic couplings. This rule was employed to give the whole hierarchy expansion layer by layer.

2 . When the vibrational period of a mode is close to the time scale of the pure electronic motion or its vibronic coupling is larger, we assumed that this mode is important to dynamics. We tried to put them in the upper branches and gave them more bases. For other unimportant modes, we tried to put them in the lower branches and gave them fewer bases.

3. We tried to run test calculations until the convergence was reached. The eigenvalues of the density matrices at each layer, the so-called natural populations, is one of parameters to indicate whether the basis number is enough. In this step, we tried to increase the number of basis functions for important branches and also for the upper branches. If the basis numbers of two child branches connecting to the same parent branch were significantly different, we took some modes from the child branch with more bases to rearrange the tree topology, and tried to reach the case that the basis numbers of all branches belonging to the same node are not significantly different. When the results did not change with increasing basis number, we assumed that the dynamics calculations were converged.

4. As a variational method, the ML-MCTDH calculations may fail to give the correct result when improper wave function expansion is used. It is very important to make sure that the optimal solution is achieved in the variational treatment. We also tried to run trees with different topologies and to compare their results. This step helped us to make sure the optimal converged result was really achieved.

Because the above testing calculations are rather timeconsuming and tedious, we developed our homemade programs (based on Perl) to run ML-MCTDH calculations, collect results, rearrange the tree topology, and increase the number of basis functions in convergence tests. If the population dynamics remains basically unchanged, we assume the convergence is reached. As we know, the ML-MCTDH expansion is not unique and the same result may be obtained from different tree expansions. Here our homemade code mainly focuses on the convergence of population dynamics, and no particular constraint was added in the evolution of tree topology except the above-mentioned principles. This indicates that we do not take into account the physical meaning of the final tree topology, if the convergence is achieved. In this sense, our approach is a practical approach that only focuses on the efficient generation of the effective and useful ML-MCTDH trees.

The Heidelberg MCTDH package requires that the subtree of each electronic state should be the same in its input file for the ML-MCTDH calculations. Thus, we show the subtree (tree A of the R-2- $\Delta_{2}-114$ model for dimer I) belongs to the single electronic state in Figure S2 in the SI. The corresponding input files of ML-MCTDH calculations are given in the SI. Two trees with different topologies (named tree B and tree C) were also built. Tree B includes several tribranches in the expansion. Tree $\mathrm{C}$ includes several tri/tetrabranches, and the mode combinations are used in the bottom layer. Tree $\mathrm{A}$, tree $\mathrm{B}$, and tree $\mathrm{C}$ give the same results (Figure S3 in the SI); thus, the convergence is fully achieved. Because three trees give identical results, we just give the input files of the calculations with tree $\mathrm{B}$ and tree $\mathrm{C}$ in the SI.

\section{ASSOCIATED CONTENT}

\section{Supporting Information}

The Supporting Information is available free of charge on the ACS Publications website at DOI: 10.1021/acs.jpcc.7b08175.

Temperature effects, typical ML-MCTDH tree structure, convergence test results, and some input files in the MLMCTDH calculations (PDF)

\section{AUTHOR INFORMATION}

\section{Corresponding Authors}

*E-mail: xieyu@qibebt.ac.cn (Y.X.).

*E-mail: yuan@qdu.edu.cn (F.Y.).

*E-mail: lanzg@qibebt.ac.cn. Fax: +86-532-80662778. Tel.: +86-532-80662630 (Z.L.).

\section{ORCID}

Yuanping Yi: 0000-0002-0052-9364

Yu Xie: 0000-0001-8925-6958

\section{Notes}

The authors declare no competing financial interest.

\section{ACKNOWLEDGMENTS}

This work was supported by NSFC projects (21673266, 21503248). This work was also supported by the Natural Science Foundation of Shandong Province for Distinguished Young Scholars (JQ201504). The authors also thank the Super Computational Centre of CAS-QIBEBT for providing computational resources. 


\section{REFERENCES}

(1) Feron, K.; Belcher, W. J.; Fell, C. J.; Dastoor, P. C. Organic Solar Cells: Understanding the Role of Forster Resonance Energy Transfer. Int. J. Mol. Sci. 2012, 13, 17019-17047.

(2) Spanggaard, H.; Krebs, F. C. A Brief History of the Development of Organic and Polymeric Photovoltaics. Sol. Energy Mater. Sol. Cells 2004, 83, 125-146

(3) Zhao, W. C.; Li, S. S.; Yao, H. F.; Zhang, S. Q.; Zhang, Y.; Yang, B.; Hou, J. H. Molecular Optimization Enables over 13\% Efficiency in Organic Solar Cells. J. Am. Chem. Soc. 2017, 139, 7148-7151.

(4) Lloyd, M. T.; Anthony, J. E.; Malliaras, G. G. Photovoltaics from Soluble Small Molecules. Mater. Today 2007, 10, 34-41.

(5) Roncali, J. Molecular Bulk Heterojunctions: An Emerging Approach to Organic Solar Cells. Acc. Chem. Res. 2009, 42, 17191730.

(6) Deng, D.; Zhang, Y. J.; Zhang, J. Q.; Wang, Z. Y.; Zhu, L. Y.; Fang, J.; Xia, B. Z.; Wang, Z.; Lu, K.; Ma, W.; Wei, Z. X. FluorinationEnabled Optimal Morphology Leads to over $11 \%$ Efficiency for Inverted Small-Molecule Organic Solar Cells. Nat. Commun. 2016, 7, 13740 .

(7) Kyaw, A. K. K.; Wang, D. H.; Luo, C.; Cao, Y.; Nguyen, T. Q.; Bazan, G. C.; Heeger, A. J. Effects of Solvent Additives on Morphology, Charge Generation, Transport, and Recombination in Solution-Processed Small-Molecule Solar Cells. Adv. Energy Mater. 2014, 4, 1301469.

(8) Kaake, L. G.; Welch, G. C.; Moses, D.; Bazan, G. C.; Heeger, A. J. Influence of Processing Additives on Charge-Transfer Time Scales and Sound Velocity in Organic Bulk Heterojunction Films. J. Phys. Chem. Lett. 2012, 3, 1253-1257.

(9) Huang, Y.; Liu, F.; Guo, X.; Zhang, W.; Gu, Y.; Zhang, J. P.; Han, C. C.; Russell, T. P.; Hou, J. H. Manipulating Backbone Structure to Enhance Low Band Gap Polymer Photovoltaic Performance. Adv. Energy Mater. 2013, 3, 930-937.

(10) Pensack, R. D.; Guo, C.; Vakhshouri, K.; Gomez, E. D.; Asbury, J. B. Influence of Acceptor Structure on Barriers to Charge Separation in Organic Photovoltaic Materials. J. Phys. Chem. C 2012, 116, 48244831.

(11) Cho, S.; Rolczynski, B. S.; Xu, T.; Yu, L. P.; Chen, L. X. Solution Phase Exciton Diffusion Dynamics of a Charge-Transfer Copolymer PTB7 and a Homopolymer P3HT. J. Phys. Chem. B 2015, 119, 74477456.

(12) Denis, J.-C.; Schumacher, S.; Hedley, G. J.; Ruseckas, A.; Morawska, P. O.; Wang, Y.; Allard, S.; Scherf, U.; Turnbull, G. A.; Samuel, I. D. W.; Galbraith, I. Subpicosecond Exciton Dynamics in Polyfluorene Films from Experiment and Microscopic Theory. J. Phys. Chem. C 2015, 119, 9734-9744.

(13) Hoffman, D. P.; Leblebici, S. Y.; Schwartzberg, A. M.; Mathies, R. A. Exciton Mobility in Organic Photovoltaic Heterojunctions from Femtosecond Stimulated Raman. J. Phys. Chem. Lett. 2015, 6, 29192923.

(14) Ayzner, A. L.; Doan, S. C.; de Villers, B. T.; Schwartz, B. J. Ultrafast Studies of Exciton Migration and Polaron Formation in Sequentially Solution-Processed Conjugated Polymer/Fullerene Quasi-Bilayer Photovoltaics. J. Phys. Chem. Lett. 2012, 3, 2281-2287.

(15) Sung, J.; Kim, P.; Fimmel, B.; Würthner, F.; Kim, D. Direct Observation of Ultrafast Coherent Exciton Dynamics in Helical PiStacks of Self-Assembled Perylene Bisimides. Nat. Commun. 2015, 6, 8646.

(16) Wang, T.; Chan, W. L. Dynamical Localization Limiting the Coherent Transport Range of Excitons in Organic Crystals. J. Phys. Chem. Lett. 2014, 5, 1812-1818.

(17) Wang, T.; Kafle, T. R.; Kattel, B.; Chan, W. L. Observation of an Ultrafast Exciton Hopping Channel in Organic Semiconducting Crystals. J. Phys. Chem. C 2016, 120, 7491-7499.

(18) Ke, Y.; Liu, Y.; Zhao, Y. Visualization of Hot Exciton Energy Relaxation from Coherent to Diffusive Regimes in Conjugated Polymers: A Theoretical Analysis. J. Phys. Chem. Lett. 2015, 6, $1741-1747$
(19) Jiang, Y.; Zhong, X.; Shi, W.; Peng, Q.; Geng, H.; Zhao, Y.; Shuai, Z. Nuclear Quantum Tunnelling and Carrier Delocalization Effects to Bridge the Gap between Hopping and Bandlike Behaviors in Organic Semiconductors. Nanoscale Horiz. 2016, 1, 53-59.

(20) Binder, R.; Polkehn, M.; Ma, T.; Burghardt, I. Ultrafast Exciton Migration in an HJ-Aggregate: Potential Surfaces and Quantum Dynamics. Chem. Phys. 2017, 482, 16-26.

(21) Schröter, M.; Ivanov, S. D.; Schulze, J.; Polyutov, S. P.; Yan, Y.; Pullerits, T.; Kühn, O. Exciton-Vibrational Coupling in the Dynamics and Spectroscopy of Frenkel Excitons in Molecular Aggregates. Phys. Rep. 2015, 567, 1-78.

(22) Zhang, H. J.; Malrieu, J. P.; Ma, H. B.; Ma, J. Implementation of Renormalized Excitonic Method at Ab Initio Level. J. Comput. Chem. 2012, 33, 34-43.

(23) Ma, Y. J.; Liu, Y.; Ma, H. B. A New Fragment-Based Approach for Calculating Electronic Excitation Energies of Large Systems. J. Chem. Phys. 2012, 136, 024113.

(24) Ma, H.; Troisi, A. Direct Optical Generation of Long-Range Charge-Transfer States in Organic Photovoltaics. Adv. Mater. 2014, 26, 6163-6167.

(25) Spano, F. C.; Silva, C. H- and J-Aggregate Behavior in Polymeric Semiconductors. Annu. Rev. Phys. Chem. 2014, 65, 477-500.

(26) Yamagata, H.; Maxwell, D. S.; Fan, J.; Kittilstved, K. R.; Briseno, A. L.; Barnes, M. D.; Spano, F. C. HJ-Aggregate Behavior of Crystalline 7,8,15,16-Tetraazaterrylene: Introducing a New Design Paradigm for Organic Materials. J. Phys. Chem. C 2014, 118, 2884228854.

(27) Häse, F.; Valleau, S.; Pyzer-Knapp, E.; Aspuru-Guzik, A. Machine Learning Exciton Dynamics. Chem. Sci. 2016, 7, 5139-5147.

(28) You, Z. Q.; Hsu, C. P. Theory and Calculation for the Electronic Coupling in Exciation Energy Transfer. Int. J. Quantum Chem. 2014, $114,102-115$.

(29) Lin, L.-Y.; Chen, Y.-H.; Huang, Z.-Y.; Lin, H.-W.; Chou, S.-H.; Lin, F.; Chen, C.-W.; Liu, Y.-H.; Wong, K.-T. A Low-Energy-Gap Organic Dye for High-Performance Small-Molecule Organic Solar Cells. J. Am. Chem. Soc. 2011, 133, 15822-15825.

(30) Chang, A. Y.; Chen, Y. H.; Lin, H. W.; Lin, L. Y.; Wong, K. T.; Schaller, R. D. Charge Carrier Dynamics of Vapor-Deposited SmallMolecule/Fullerene Organic Solar Cells. J. Am. Chem. Soc. 2013, 135, 8790-8793.

(31) Shen, X. X.; Han, G. C.; Yi, Y. P. Multiscale Description of Molecular Packing and Electronic Processes in Small-Molecule Organic Solar Cells. Chin. Chem. Lett. 2016, 27, 1453-1463.

(32) Shen, X. X.; Han, G. C.; Yi, Y. P. The nature of excited states in dipolar donor/fullerene complexes for organic solar cells: evolution with the donor stack size. Phys. Chem. Chem. Phys. 2016, 18, 1595515963.

(33) Han, G.; Shen, X.; Yi, Y. Deposition Growth and Morphologies of C60 on DTDCTB Surfaces: An Atomistic Insight into the Integrated Impact of Surface Stability, Landscape, and Molecular Orientation. Adv. Mater. Interfaces 2015, 2, 1500329.

(34) Shen, X.; Han, G.; Fan, D.; Xie, Y.; Yi, Y. Hot Charge-Transfer States Determine Exciton Dissociation in the DTDCTB/C60 Complex for Organic Solar Cells: A Theoretical Insight. J. Phys. Chem. C 2015, 119, 11320-11326.

(35) Wang, H. B.; Thoss, M.; Miller, W. H. Systematic Convergence in the Dynamical Hybrid Approach for Complex Systems: A Numerically Exact Methodology. J. Chem. Phys. 2001, 115, 29792990.

(36) Thoss, M.; Wang, H. B.; Miller, W. H. Self-Consistent Hybrid Approach for Complex Systems: Application to the Spin-Boson Model with Debye Spectral Density. J. Chem. Phys. 2001, 115, 2991-3005.

(37) Wang, H.; Thoss, M. A Multilayer Multiconfiguration TimeDependent Hartree Simulation of the Reaction-Coordinate SpinBoson Model Employing an Interaction Picture. J. Chem. Phys. 2017, 146,124112

(38) Tamura, H.; Burghardt, I.; Tsukada, M. Exciton Dissociation at Thiophene/Fullerene Interfaces: The Electronic Structures and Quantum Dynamics. J. Phys. Chem. C 2011, 115, 10205-10210. 
(39) Tamura, H.; Martinazzo, R.; Ruckenbauer, M.; Burghardt, I. Quantum Dynamics of Ultrafast Charge Transfer at an Oligothiophene-Fullerene Heterojunction. J. Chem. Phys. 2012, 137, 22 A540.

(40) Tamura, H.; Tsukada, M. Role of Intermolecular Charge Delocalization on Electron Transport in Fullerene Aggregates. Phys. Rev. B: Condens. Matter Mater. Phys. 2012, 85, 054301.

(41) Tamura, H.; Burghardt, I. Ultrafast Charge Separation in Organic Photovoltaics Enhanced by Charge Delocalization and Vibronically Hot Exciton Dissociation. J. Am. Chem. Soc. 2013, 135, 16364-16367.

(42) Tamura, H.; Burghardt, I. Potential Barrier and Excess Energy for Electron-Hole Separation from the Charge-Transfer Exciton at Donor-Acceptor Heterojunctions of Organic Solar Cells. J. Phys. Chem. C 2013, 117, 15020-15025.

(43) Huix-Rotllant, M.; Tamura, H.; Burghardt, I. Concurrent Effects of Delocalization and Internal Conversion Tune Charge Separation at Regioregular Polythiophene-Fullerene Heterojunctions. J. Phys. Chem. Lett. 2015, 6, 1702-1708.

(44) Tamura, H. Diabatization for Time-Dependent Density Functional Theory: Exciton Transfers and Related Conical Intersections. J. Phys. Chem. A 2016, 120, 9341-9347.

(45) Martinazzo, R.; Hughes, K. H.; Martelli, F.; Burghardt, I. Effective Spectral Densities for System-Environment Dynamics at Conical Intersections: S2-S1 Conical Intersection in Pyrazine. Chem. Phys. 2010, 377, 21-29.

(46) Wang, H.; Thoss, M. From Coherent Motion to Localization: Dynamics of the Spin-Boson Model at Zero Temperature. New J. Phys. 2008, 10, 115005 .

(47) Weiss, U. Quantum Dissipative Systems; World Scientific: 2008.

(48) Leggett, A. J.; Chakravarty, S.; Dorsey, A. T.; Fisher, M. P. A.; Garg, A.; Zwerger, W. Dynamics of the Dissipative 2-State System. Rev. Mod. Phys. 1987, 59, 1-85.

(49) Meyer, H. D.; Worth, G. A. Quantum Molecular Dynamics: Propagating Wavepackets and Density Operators Using the Multiconfiguration Time-Dependent Hartree Method. Theor. Chem. Acc. 2003, 109, 251-267.

(50) Manthe, U. The Multi-Configurational Time-Dependent Hartree Approach Revisited. J. Chem. Phys. 2015, 142, 244109.

(51) Meng, Q.; Faraji, S.; Vendrell, O.; Meyer, H.-D. Full Dimensional Quantum-Mechanical Simulations for the Vibronic Dynamics of Difluorobenzene Radical Cation Isomers Using the Multilayer Multiconfiguration Time-Dependent Hartree Method. J. Chem. Phys. 2012, 137, 134302.

(52) Meng, Q.; Meyer, H.-D. A Multilayer MCTDH Study on the Full Dimensional Vibronic Dynamics of Naphthalene and Anthracene Cations. J. Chem. Phys. 2013, 138, 014313.

(53) Vendrell, O.; Meyer, H.-D. Multilayer Multiconfiguration TimeDependent Hartree Method: Implementation and Applications to a Henon-Heiles Hamiltonian and to Pyrazine. J. Chem. Phys. 2011, 134, 044135 .

(54) Wang, H. B.; Thoss, M. Multilayer Formulation of the Multiconfiguration Time-Dependent Hartree Theory. J. Chem. Phys. 2003, 119, 1289-1299.

(55) Manthe, U. A Multilayer Multiconfigurational Time-Dependent Hartree Approach for Quantum Dynamics on General Potential Energy Surfaces. J. Chem. Phys. 2008, 128, 164116.

(56) Manthe, U. Layered Discrete Variable Representations and Their Application within the Multiconfigurational Time-Dependent Hartree Approach. J. Chem. Phys. 2009, 130, 054109.

(57) Vendrell, O.; Meyer, H.-D. The MCTDH Package, version 8.5; 2011. http://mctdh.uni-hd.de.

(58) Schröter, M.; Kühn, O. Interplay Between Nonadiabatic Dynamics and Frenkel Exciton Transfer in Molecular Aggregates: Formulation and Application to a Perylene Bismide Model. J. Phys. Chem. A 2013, 117, 7580-7588.

(59) Xie, Y.; Zheng, J.; Lan, Z. Full-Dimensional Multilayer Multiconfigurational Time-Dependent Hartree Study of Electron Transfer Dynamics in the Anthracene/C60 Complex. J. Chem. Phys. 2015, 142, 084706.
(60) Zheng, J.; Xie, Y.; Jiang, S.; Lan, Z. Ultrafast Nonadiabatic Dynamics of Singlet Fission: Quantum Dynamics with the Multilayer Multiconfigurational Time-Dependent Hartree (ML-MCTDH) Method. J. Phys. Chem. C 2016, 120, 1375-1389. 